Phenotypic characterization of the Epha4-inversion embryos revealed polydactyly in Inv1 and Inv2 mutants, which the authors attribute to early activation of $I h h$ expression (which normally does not occur before E12.5). Studying the $3 \mathrm{D}$ chromatin context, the inversions were found to lead to asymmetric chromatin contacts around the breakpoints that presented as a stripe-like pattern in the cHi-C maps, or 'architectural stripes'. The inverted CTCF motifs were found at the base of the stripe, and genes located under the stripe were upregulated in Inv1 mutant embryos, in accordance with the notion that orientation of CTCF sites influences loop formation. When CTCF sites were deleted in Inv1, architectural stripes were lost in this region.

Unlike in limb buds, the Epha4 locus is inactive in mouse embryonic stem cells (mESCs). To delineate the role of enhancer activity in stripe formation, Inv1-mutant mESCs were analysed. Chromatin structure at the locus was observed that differed from that seen in limb buds, showing much

By computationally generating pairwise contacts from ChIA-Drop data, the authors found that the patterns of chromatin structure were comparable between $\mathrm{Hi}-\mathrm{C}$ and ChIA-Drop. However, in contrast to $\mathrm{Hi}-\mathrm{C}$, ChIA-Drop was able to resolve the multiplex nature of chromatin interactions, inside and between TADs, at the single-molecule level, identifying a high degree of heterogeneity.

The authors found that most ChIADrop TADs were associated with repressed chromatin. By contrast, the gap regions around TAD boundaries were largely transcriptionally active. The architectural proteins BEAF-32 and $\mathrm{Su}(\mathrm{Hw})$ were shown to bind predominantly to TAD boundaries or within TADs, respectively, leading the authors to hypothesize a role in chromatin compaction.

To analyse the multiplex chromatin interactions involved in transcriptional regulation, the authors applied the RNAPII ChIA-Drop method to S2 cells. While the chromatin organizational features revealed by RNAPII ChIA-Drop resembled those identified by ChIA-Drop, a markedly reduced signal in repressed domains (that is, TADs) and weaker stripe formation (although cHi-C maps did not show perfect symmetry, indicating that enhancer activity alone is not sufficient for stripe formation).

To determine whether the architectural stripe-active enhancer relationship is a general phenomenon in the developing mouse limb bud, normalized $\mathrm{Hi}-\mathrm{C}$ data were screened across the whole genome. Active enhancer marks were found to aggregate under stripes, whereas other chromatin marks showed a more diffuse distribution, lending further evidence to the hypothesis that enhancer activity contributes to stripe formation.

This study highlights the importance of architectural stripes and their association with enhancer activity during development.

Michelle Trenkmann, Associate Editor, Nature Communications

ORIGINAL ARTICLE Kraft, K. et al. Serial genomic inversions induce tissue-specific architectural stripes, gene misexpression and congenital malformations. Nat. Cell Biol. https://doi.org/ 10.1038/s41556-019-0273-x (2019) FURTHER READING Spielmann, M. et al. Structural variation in the 3D genome. Nat. Rev. Genet. 19 , 453-467 (2018)

enrichment in transcriptionally active boundary regions was observed for RNAPII ChIA-Drop structures.

Focusing on complexes in RNAPIIassociated interaction domains that involve promoters, the team found that the majority $(80 \%)$ involve only one promoter. This finding suggests that, although some chromatin complexes involve two or more promoters, promoter-promoter interactions are less common than previously supposed. RNAPII ChIA-Drop complexes involving promoters also showed a strong directionality bias into the gene body, supporting a loop extrusion model of transcription.

In the future, complementing population-level analyses based on aggregated pairwise contacts with the single-molecule view of complex chromatin contacts provided by ChIA-Drop may strengthen novel insights or help resolve outstanding controversies.

Linda Koch

ORIGINAL ARTICLE Zheng, M. et al. Multiplex chromatin interactions with single-molecule precision. Nature https://doi.org/10.1038/ s41586-019-0949-1 (2019)

\section{GENOME EDITING}

\section{A new Cas in town}

A study in Nature reports the organization and mechanism of action of CasX, a recently identified RNA-guided DNA endonuclease that can be used for genome editing in human cells. This enzyme family seems to be functionally distinct to known CRISPR-Cas genome editors and offers exploitable advantages over existing systems, including its small size, high guide RNA content and minimal trans cleavage activity.

The RNA-guided proteins Cas9 and Cas12a form part of the archaeal and bacterial adaptive immune system CRISPR, which protects against invading nucleic acids. These endonucleases have been repurposed as 'molecular scissors', that is, genome editing tools that can be directed to cut designated genomic locations across a range of cell types and organisms. However, existing CRISPR-Cas systems have limitations to their applicability, fuelling the search for alternative enzymes.

A previous metagenomic analysis of groundwater samples identified the protein CasX, which was found to disrupt bacterial transformation by plasmid DNA through an unknown mechanism when expressed together with RNA complementary to the plasmid. This finding suggested a probable function as a DNA endonuclease despite little sequence similarity with known Cas proteins.

Liu, Orlova, Oakes et al. combined purified wild-type CasX from Deltaproteobacteria (DpbCasX) with a single guide RNA and observed that, in vitro, Cas X cleaves DNA sequences complementary to a 20-nucleotide segment of the guide RNA by generating a staggered DNA double-strand break.

To determine whether CasX can effectively manipulate genomes in vivo, the team expressed DpbCasX in Escherichia coli using a guide RNA complementary to an integrated reporter, which led to reduced cell viability in a cleavage assay. Moreover, a nuclease-deactivated DpbCasX, combined with guide RNAs targeting different sites within a reporter gene, was able to silence GFP expression in E. coli, indicating the suitability of using CasX for CRISPR interference (CRISPRi). Furthermore, catalytically active CasX was capable of inducing DNA double-strand breaks and gene editing in HEK293T cells, albeit with limited efficiency. A CasX from Planctomycetes (PlmCasX), which exhibits $\sim 70 \%$ sequence identity to DpbCasX, showed higher efficiency than DpbCasX.

Structural data from cryo-electron microscopy (cryo-EM) images showed that the guide RNA forms a prominent scaffold that dominates the architecture of the enzyme. Taken together, this study suggests that Cas $X$ will be a useful addition to the armamentarium of RNA-programmed genome-editing platforms.

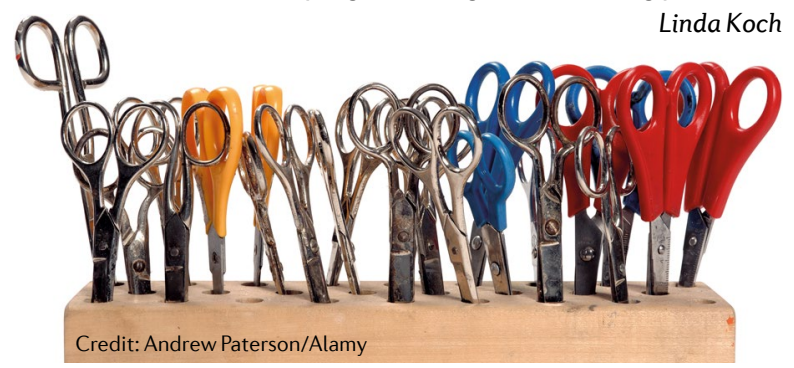

ORIGINAL ARTICLE Liu, J.-J., Orlova, N., Oakes, B. L. et al. CasX enzymes comprise a distinct family of RNA-guided genome editors. Nature https://doi.org/10.1038/s41586019-0908-x(2019) 